\title{
Magnetic field-driven superconductor-insulator transition in boron-doped nanocrystalline chemical vapor deposition diamond
}

\author{
Gufei Zhang, ${ }^{1, a)}$ J. Vanacken, ${ }^{1}$ J. Van de Vondel, ${ }^{1}$ W. Decelle, ${ }^{1}$ J. Fritzsche, ${ }^{1}$ \\ V. V. Moshchalkov, ${ }^{1}$ B. L. Willems, ${ }^{2}$ S. D. Janssens, ${ }^{3}$ K. Haenen, ${ }^{3,4}$ and P. Wagner ${ }^{3,4}$ \\ ${ }_{1}^{1}$ INPAC-Insititute for Nanoscale Physics and Chemistry, Katholieke Universiteit Leuven, \\ Celestijnenlaan 200D, B-3001 Leuven, Belgium \\ ${ }^{2}$ Facultad de Ciencias Fisicas, Universidad Nacional Mayor de San Marcos, P.O. Box 14-0149, Lima 14, \\ Peru \\ ${ }^{3}$ Institute for Materials Research (IMO), Hasselt University, Wetenschapspark 1, B-3590 Diepenbeek, \\ Belgium \\ ${ }^{4}$ Division IMOMEC, IMEC vzw, Wetenschapspark 1, B-3590 Diepenbeek, Belgium
}

(Received 31 March 2010; accepted 5 May 2010; published online 7 July 2010)

\begin{abstract}
The systematics of the suppression of superconductivity with increasing magnetic field in boron-doped nanocrystalline chemical vapor deposition diamond is studied in a broad temperature range. At the temperature of $T_{\mathrm{S} 0}$ which is above the critical temperature, a plateau is observed in the resistivity versus temperature curve $\rho(T)$ taken at zero magnetic field. When a magnetic field of $B=B_{S N}(N=1,2, \ldots, 5)$ is applied, the plateau moves to low temperature with the thermoresistivity maximum located at $T_{S N}(N=1,2, \ldots, 5)$. The $\rho(B)$ curves, measured at different temperatures around $T_{S N}$, intersect in the $\rho$-B plane at the field of $B=B_{S N}$. By tuning $B_{S N}$ from 0 to $5 \mathrm{~T}$, a series of plateaus in the $\rho$-T plane and the corresponding intersections in the $\rho$-B plane are observed. The intersections quadratically chain up in the $\rho$-B plane, separating the superconducting from the insulating region. The thermoresistivity maxima exponentially group up in the $\rho$ - $\mathrm{T}$ plane, thus defining a phase fluctuation zone. The phase boundary, composed of the intersections and separating the superconducting states from the insulating state, is shown to be a generic consequence of granularity. (C) 2010 American Institute of Physics. [doi:10.1063/1.3437653]
\end{abstract}

\section{INTRODUCTION}

Diamond, one of the most revered and sought-after materials by humankind, is taking on a whole new industrial value owing to its extraordinary mechanical toughness, uniquely high thermal conductivity, and tunable electrical properties. ${ }^{1}$ More than a featureless wide-gapped insulator, small concentrations of boron can transform diamonds into doped semiconductors, and at a certain critical doping level, boron-doped diamonds (BDDs) undergo an insulator-metal transition. All these physical properties make BDD a promising candidate to boost the Si-based technologies for the next generation of more robust, faster, higher voltage electronic devices. ${ }^{2}$ In addition, type-II superconductivity has recently been reported for heavily boron-doped bulk ${ }^{3}$ and thin film ${ }^{4}$ polycrystalline diamonds, providing even more fascinating possibilities of the integration of semiconductor and superconductor electronics.

For the potential applications of the BDDs, it is crucial to synthesize diamond in the form of wafers/thin films so that it can be printed with circuit boards and shaped into other high-tech devices. Chemical vapor deposition (CVD), a method widely used in the semiconductor industry, is being employed to produce high-purity BDDs, offering opportunities for their practical uses ${ }^{5}$ and providing stages for fundamental research on superconductivity. ${ }^{4,6-13}$ Although important knowledge has been accumulated concerning the

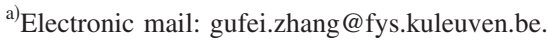

properties of boron-doped CVD diamond thin films, ${ }^{4,6-9,11,12}$ only a few research reports have been devoted to the granular nature of this superconductivity. The influence of the inevitable granularity of CVD diamond thin films on their transport properties was only lately brought to attention. ${ }^{13,14}$ In this article, we present results concerning the temperatureand magnetic field-driven superconductor-insulator transitions (SITs) in nanocrystalline boron-doped CVD diamond thin films. We have observed negative thermoresistivity (NTR) in the $\rho$-T plane, and with help of this observation, in the $\rho$-B plane, detected a series of intersections of the magnetoresistivity curves taken within well defined temperature windows. The intersections, a consequence of the granularity, separate the superconducting region from the insulating region.

\section{EXPERIMENT}

The BDD granular thin films were prepared by microwave plasma-enhanced CVD at Hasselt University. ${ }^{12,15}$ The sample preparation process consists of two main steps, i.e., (1) substrate seeding with a cap monolayer of nanodiamond powders $(\varnothing \sim 5-10 \mathrm{~nm})$ and (2) BDD CVD film growth on the seeding layer. (1) A substrate of quartz rather than Si was utilized for diamond deposition to avoid the formation of leakage channels at relatively high temperatures. ${ }^{16}$ The seeding-generated nucleation sites are with a density of $10^{11} \mathrm{~cm}^{-2}$, and evenly spaced with a tight intergrain distance distribution. $^{15}$ (2) The seeded substrate was placed into a steel type ASTeX 6500 reactor in combination with a con- 


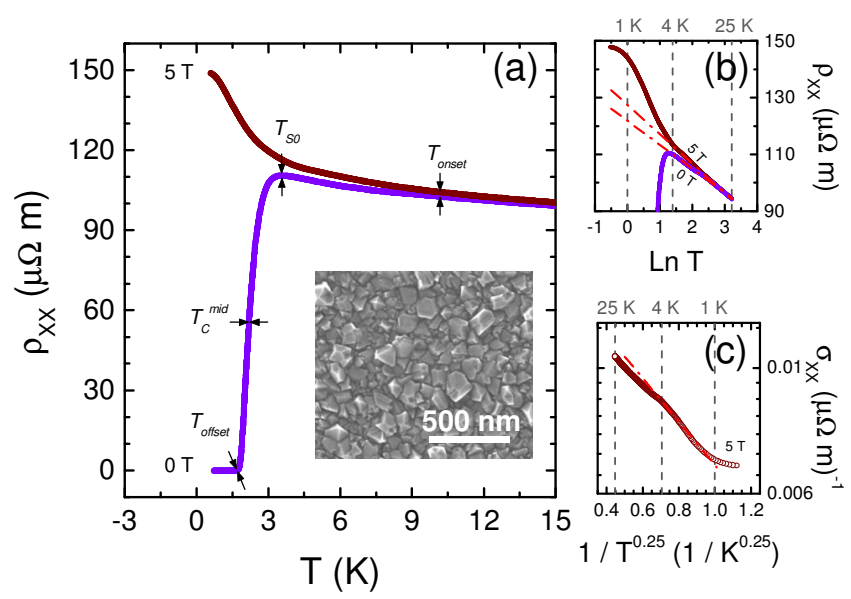

FIG. 1. (Color online) Temperature dependence of the longitudinal resistivity at several constant magnetic fields. (a) Phase fluctuation zone in between $\left.\rho_{x x}(T)\right|_{B=5 \mathrm{~T}}$ and $\left.\rho_{x x}(T)\right|_{B=0 \mathrm{~T}}$. Pair arrow-indicated characteristic temperatures: the offset temperature $T_{\text {offset }}$, the onset temperature $T_{\text {onset }}$, the critical temperature $T_{C}^{\text {mid }}$, and the temperature at the resistivity maximum $T_{S 0}$. Inset: the granular morphology characterized by SEM. (b) Plots of the resistivity vs $\operatorname{Ln} T$ for $\left.\rho_{x x}(T)\right|_{B=5 \mathrm{~T}}$ and $\left.\rho_{x x}(T)\right|_{B=0 \mathrm{~T}}$. The dotted-dashed red lines are the expected logarithmic behavior. (c) The low temperature segment (4-1 K) of the $\left.\rho_{x x}(T)\right|_{B=5 \text { T }}$ curve characterized by the FHM. The reverse resistivity is plotted vs $1 / T^{0.25}$ on a semilogarithmic scale. The dotted-dashed red line is the expected Mott's law in three dimensions.

ventional $\mathrm{CH}_{4} / \mathrm{H}_{2}$ gas mixture. Under irradiation with microwaves, the gases were agitated and a cloud of energetic carbon atoms was consequently released. These carbon atoms settled on the seeds and gradually, the growing diamond grains bordered each other. During the growth process, boron doping was achieved by trimethylboron gas injection with a $\mathrm{TMB} / \mathrm{CH}_{4}$ ratio of $8000 \mathrm{ppm}$. The average film thickness was around $83 \pm 5 \mathrm{~nm}$ for a total deposition time of $10 \mathrm{~min}$.

The granular morphology of the BDD film was characterized by scanning electron microscopy (SEM), giving a typical grain size of $90 \mathrm{~nm}$. The polycrystalline nature of the films was also identified by $x$-ray diffraction. The BDD film was patterned into a bridge $\left(3 \times 1 \mathrm{~mm}^{2}\right)$ with six terminals for transport measurements at INPAC, K. U. Leuven. Magnetoresistivity and thermoresistivity in the temperature range from $0.6 \mathrm{~K}$ to $7 \mathrm{~K}$ were studied in a Heliox ${ }^{3} \mathrm{He}$ cryostat from Oxford Instruments, equipped with a $5 \mathrm{~T}$ dc magnet. The applied magnetic fields were perpendicular to the film surface. Zero field thermoresistivity in the temperature range of $1.8-160 \mathrm{~K}$ was probed in a ${ }^{4} \mathrm{He}$ cryostat. Between 1.8 and $7 \mathrm{~K}$, the zero field thermoresistivity, measured in the two different cryostats, perfectly overlaps with each other.

\section{RESULTS AND DISCUSSION}

\section{A. Thermoresistivity and the hopping threshold}

Figure 1 shows the temperature dependence of the resistivity at a series of magnetic fields, presenting the systematic suppression of the superconductivity at low temperature. The $\rho(T)$ curves, taken at zero field and $5 \mathrm{~T}$, are plotted together in Fig. 1(a) to indicate the superconducting fluctuation zone in between. ${ }^{17-20}$ The characteristic temperatures, i.e., the offset temperature $T_{\text {offset }}=1.67 \mathrm{~K}$, the onset temperature $T_{\text {onset }}$ $\sim 10.16 \mathrm{~K}$, the critical temperature $T_{C}^{\text {mid }}=2.20 \mathrm{~K}$, and the temperature at the zero field resistivity maximum $T_{\mathrm{S} 0}$ $=3.55 \mathrm{~K}$, are labeled with arrows. The $\left.\rho_{x x}(T)\right|_{B=5 \mathrm{~T}}$ curve shows a significant NTR with $\rho_{x x}$ variations per decade temperature of $\left.\Delta \rho_{x x}\right|_{\text {per } 10 \mathrm{~K}}=90.6 \%$ over the temperature range of $1-4 \mathrm{~K}$ and $\left.\Delta \rho_{x x}\right|_{\text {per } 10 \mathrm{~K}}=21.9 \%$ over the temperature range of $1-25 \mathrm{~K}$. Such strong $\rho_{x x}$ variations cannot be taken as signature of a typical metallic behavior. ${ }^{21}$ Moreover, as shown in Fig. 1(b), the $\left.\rho_{x x}(T)\right|_{B=5}$ T curve deviates distinctly from the linear relationship between $\rho_{x x}$ and $\ln (T)$ below $4 \mathrm{~K}$.

The origin of the logarithmic temperature-dependence of $\rho_{x x}$ between 25 and $4 \mathrm{~K}$ still remains unknown while it definitely should not be ascribed to weak localization effect, since for which a $\left.\Delta \rho_{x x}\right|_{\text {per } 10 \mathrm{~K}}<10 \%$ is normally expected. ${ }^{21}$ Between 4 and $1 \mathrm{~K}$, the deviation from the logarithmic behavior is found to be exponential. Therefore, the fermion hopping mechanisms (FHMs) could be taken into account. ${ }^{21}$ For granular systems, the FHMs have been explained based on a physical model of thermally activated tunneling between grains with different charging energy. ${ }^{22-25}$ The transport properties of our granular BDD sample with dispersive size distribution of grains and different intergrain spacings support such a physical picture. ${ }^{21}$ By writing $\sigma(T)=\sigma_{0} \exp \left\{-\left(T_{0} / T\right)^{\alpha}\right\}$ with $\alpha=1$ for the next neighbor hopping, $\alpha=1 / 2$ for the Shklovskii-Efros law, ${ }^{26}$ and $\alpha$ $=1 / 4$ for the variable range hopping (VRH, Mott's law in three dimensions), ${ }^{27}$ the FHM characterization can be carried out. As shown in Fig. 1(c), plotting the reverse resistivity of the $\left.\rho_{x x}(T)\right|_{B=5 \text { T }}$ curve as a function of $T^{-0.25}$ on a semilogarithmic scale, the low temperature segment $(1-4 \mathrm{~K})$ of the curve is found to be in good agreement with the VRH model $(\alpha=0.25$, Mott's law in three dimensions). It should be, however, noted that the temperature interval $1-4 \mathrm{~K}$ is rather narrow. The slope of the linear VRH fitting on the semilogarithmic scale is given by $-T_{0}^{\alpha} \cdot \log _{10}(e)$ with $T_{0}$ being a measure of the quasiparticle correlation strength. ${ }^{28}$ The value of $T_{0}$ obtained by the fitting is $0.65 \mathrm{~K}$, far smaller than $T_{0}=46 \mathrm{~K}$ for Sato's semiconducting BDD in the temperature range of $100-300 \mathrm{~K}$ (VRH, Mott's law in three dimensions ${ }^{29}$ and $T_{0}=3700 \mathrm{~K}$ for Klein's insulating BDD in the temperature range of $10-300 \mathrm{~K}$ (VRH, Mott's law in three dimensions). ${ }^{9}$ Since the localization radius $a_{H}$ of boron is related to $T_{0}$ through $a_{H} \sim T_{0}^{\alpha / \alpha-1}, 28$ the small-valued $T_{0}$ suggests a much larger $a_{H}$ value based on $a_{H} \sim T_{0}^{-1 / 3}$ in our case.

The exponential increase in $\left.\rho_{x x}(T)\right|_{B=5} \mathrm{~T}$ fades away around $1 \mathrm{~K}$ below which the remanent superconductivity is bending the curve over. In studying the zero field temperature dependence of the longitudinal resistivity, evidenced by the linear fit to the $\left.\rho_{x x}(T)\right|_{B=0 \text { T }}$ curve in the $\rho_{x x}-\operatorname{Ln} T$ plane, within the temperature range of $25 \mathrm{~K}>T>T_{\mathrm{S} 0}$, the sample is still nonmetallic, although the increase in the resistivity no longer obeys an exponential law. ${ }^{21}$ By now, it is clear that at low temperatures, a sufficiently high magnetic field can trigger the exponential increase in the sample resistivity (the insulating state). Such a global insulating state may be due to the destruction of intergrain superconducting coherence by the magnetic field. In our case, obviously, this coherence suppression overcomes the competing mechanism of quasiparticle tunneling between the grains. ${ }^{30,31}$ 

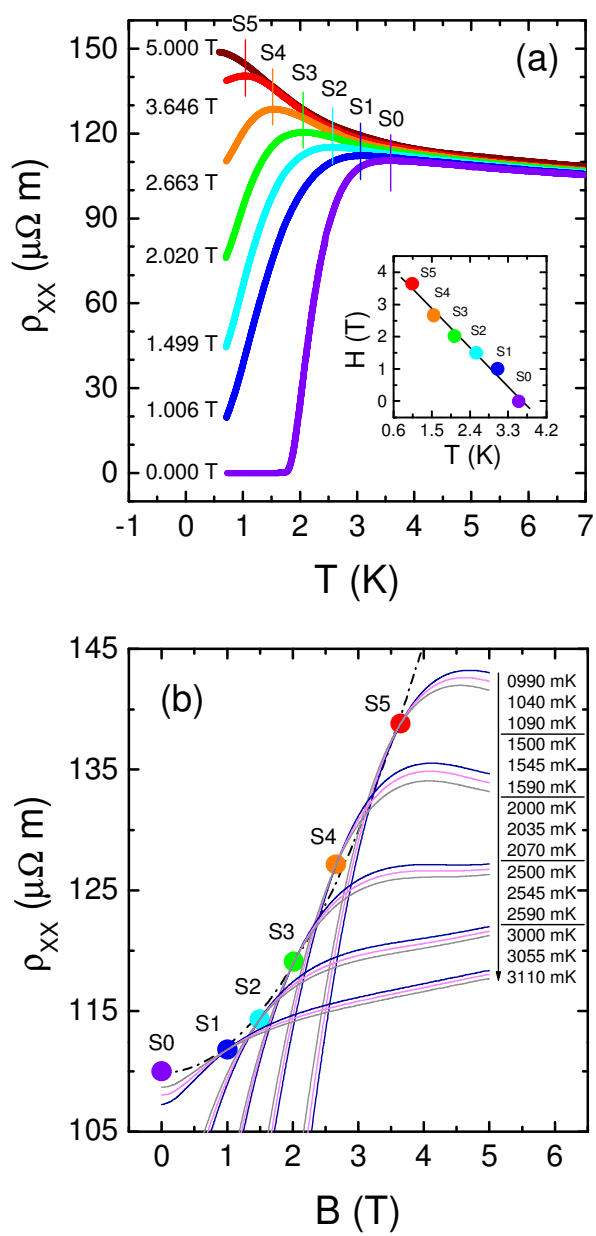

FIG. 2. (Color online) Magnetic field-driven SITs. (a) Moving of the hopping threshold by magnetic fields to the resistivity maxima as indicated by the vertical bars $(S N, N=0-5)$. Inset: the magnetic field of $S N$ plotted as a function of its temperature. (b) Magnetic field dependence of the longitudinal resistivity near and at the temperatures of $S N$. The intersections are chained by a quadratic fitting (dashed-dotted line).

As one of the important characteristic temperatures, the temperature of the resistivity maximum $T_{\mathrm{S} 0}$ is not fixed but is shifted by magnetic field. Figure 2(a) shows the magnetic field dependence of this shift. As the magnetic field $B_{S N}$ $(N=1,2, \ldots, 5)$ increases from 0 to $\sim 5 \mathrm{~T}$, the resistivity maximum shifts from $T_{\mathrm{S} 0}=3.55$ to $\sim 0 \mathrm{~K}$. As shown by the colored bars, the states of the system at the resistivity maxima are indicated and numbered in a format of " $S N$ " $(N=0 \sim 5)$. For $B_{S N} \geq 5 \mathrm{~T}$, the shift is beyond our temperature range of the measurements. Nevertheless, from the linear fit going through the states of S0 $\sim \mathrm{S} 5$ in the H-T plane [see inset of Fig. 2(a)], the critical field at zero temperature $H_{C}(0 \mathrm{~K})$ can be estimated. Based on the linear fit, at $H_{C}(0 \mathrm{~K})=4.98 \mathrm{~T}$, the last resistivity maximum of the series should be located, representing the full suppression of the superconductivity. This deduction is, however, not an evident fact, owing to the fade away of the exponential increase of $\left.\rho_{x x}(T)\right|_{B=5 \mathrm{~T}}$ below $1 \mathrm{~K}$ [see Figs. 1(b) and 1(c)]. With $H_{C}(0 \mathrm{~K})=4.98 \mathrm{~T}$ and the relation of $\xi_{\mathrm{GL}}$ $=\left[\Phi_{0} / 2 \pi H_{C}(0 \mathrm{~K})\right]^{1 / 2}$, a rough estimation of the GinzburgLaudau coherence length $\xi_{\mathrm{GL}}=8 \mathrm{~nm}$ can still be made $\left[\xi_{\mathrm{GL}}\right.$ $=10 \mathrm{~nm}$ for Ekimov's bulk BDD (Ref. 3)]. Since $\xi_{\mathrm{GL}} \ll$ the film thickness $(d=83 \mathrm{~nm})$, the sample can be considered as three dimensional (concerning its superconducting properties), which is in agreement with our FHM characterization (VRH, Mott's law in three dimensions).

\section{B. $\rho(B)$ intersections at the $S N(N=1,2, \ldots, 5)$ states}

The $S N(N=1,2, \ldots, 5)$ states separate the NTR from the PTR (positive thermoresistivity) in the $\rho$-T plane for $B_{S N}$ $\leq 3.646 \mathrm{~T}$. This feature remains in the $\rho$-B plane. As shown in Fig. 2(b), at the temperature of a $S N$ state, e.g., $T_{\mathrm{S} 5}$ $=1040 \mathrm{mK}$ for S5, we picked a narrow temperature window, e.g., $1040 \mathrm{mK} \pm 50 \mathrm{mK}$ for $\mathrm{S} 5$, within which three $\rho(B)$ curves were taken, e.g., $\rho(B)$ taken at 990, 1040, and 1090 $\mathrm{mK}$ for S5. Similar to the case in the $\rho$-T plane, at the superconducting side (left side) of the $S N$ state, e.g., S5, the $\rho(B)$ curves lie on top of each other in order of their PTR, while at the insulating side (right side) of the state, the $\rho(B)$ curves are laid in order of NTR. The change in the thermal ordering occurs right at the $S N$ state, which results in the intersection of the $\rho(B)$ curves at $B_{S N}$.

The systematically detected intersections, chained by a quadratic fitting (the dashed-dotted line), represent a phase boundary which folds the isothermal $\rho(B)$ curves coming from the insulating side down to the superconducting side. Since the transition from PTR to NTR is a characteristic of the superconductors with either intrinsic or extrinsic granularity, ${ }^{21-36}$ the intersection-chained phase boundary cannot stay free from the granularity and thus, it may be termed the granularity-specific phase boundary (GSPB). It is conceivable that for the $\rho(B)$ measurements at continuous temperatures, an insulating belt coming from high magnetic field will be folded to the superconducting region at low magnetic field and the GSPB is the very folding line of this belt. Moreover, this GSPB separates all the possible states from the top left area in the $\rho$-B plane. Therefore, a forbidden zone in the $\rho$-B plane can be defined for granular systems.

The magnetic field-driven SITs have been extensively studied for two decades. ${ }^{32-44}$ Analyzing the coincidentally detected crossover by Fisher's finite-size scaling law $^{37,45}$ is a well-known exploratory method..$^{32,35,36,40-44}$ Naturally, a bunch of isothermal $\rho(B)$ curves crossing each other, indicates the temperature independence of the magnetoresistivity at the crossover point. However, the significance of some accidentally detected crossovers has so far been overstated. The temperature independence of the magnetoresistivity was intuitively extended to $\sim 0 \mathrm{~K}$ without detailed survey. $32,34-36,39,41$

Such visually unique crossovers have always a sheet resistance noncomparable with the quantum resistance $R_{Q}$ $=6.5 \mathrm{k} \Omega / \square$ which was predicted to be universal at the quantum phase transitions $(T=0 \mathrm{~K})$ where both vortices and Cooper pairs are mobile. ${ }^{45}$ Scaling analysis of these crossovers yields various values of the scaling exponent product $\nu z$ with $\nu$ being the coherence length exponent for $\xi \sim \mid B$ $-B_{\text {cross }}{ }^{-\nu}$, and $z$ being the characteristic dynamical exponent for $\Omega \sim \xi^{-z}$, where $\left|B-B_{\text {cross }}\right|$ is the deviation from the critical point. Hereby, we emphasize the difference between the theoretical unique crossover (TUC) and our experimental nonu- 


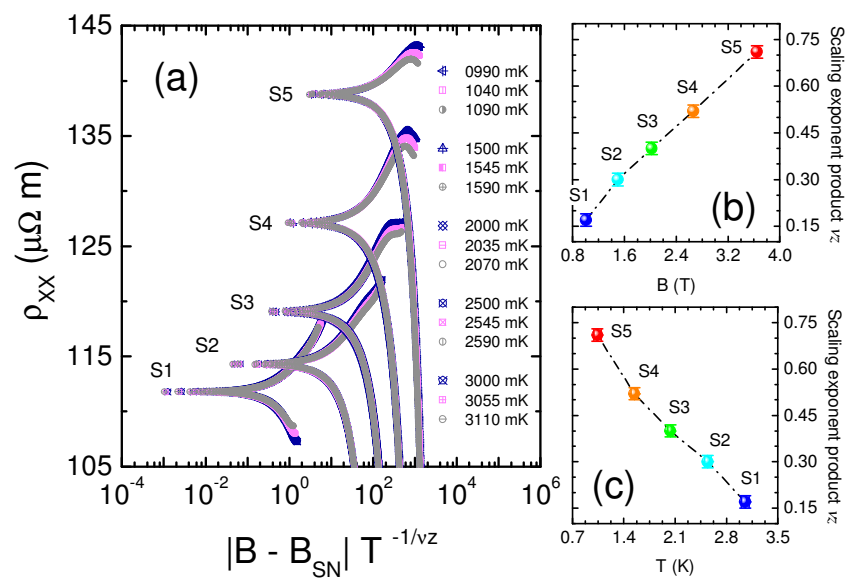

FIG. 3. (Color online) Scaling analysis of the $S N$ intersections [see Fig. 2(b)] by Fisher's finite-size scaling law, which gives various values of the scaling exponent product $\nu z$. (a) Scaling analysis carried out by the so-called optimal collapse. (b) Magnetic field dependence of the $\nu z$ value. (c) Temperature dependence of the $\nu z$ value.

nique intersections (ENI). The temperature-independent magnetoresistivity of the former is a long-range (temperature) behavior which can be virtually extended to $\sim 0 \mathrm{~K}$, while the temperature-independent magnetoresistivity of the latter is a short-range behavior (temperature) which only visually holds around $T_{S N}$ of the $S N$ state, attributed to $d \rho_{x x} /\left.d T\right|_{T_{S N}}=0$. Even so, owing to the crucial positions of the intersections in the $\rho$-T/B plane and considering the mathematical expression of the temperature-independence of the magnetoresistivity in Fisher's scaling law, $\rho /\left.\rho_{\text {cross }}\right|_{B_{\text {cross }}}$ $=\left.f\left(\left|B-B_{\text {cross }}\right| \cdot T^{-1 / \nu z}\right)\right|_{B_{\text {cross }}}=1,{ }^{37}$ a scaling analysis of the ENIs might still shed some light on the underlying physics of the magnetic field-driven SITs in granular systems, even though the physical picture of the scaling law cannot be borrowed straightforwardly.

An analytic approach to apply this scaling law is the so-called optimal collapse. ${ }^{32,42}$ Figure 3(a) demonstrates the collapses of the resistivity data as a function of $\left|B-B_{\text {cross }}\right| t$ with $t=T^{-1 / v z}$ and $B_{\text {cross }}$ being replaced by the magnetic field $B_{S N}$ at the ENIs. By assigning proper values to $t$, the $\rho_{x x}(B)$ curves collapse perfectly onto each other at the resistivity of the ENIs. In the collapsing process, the contribution of $\Delta B$ $=\left|B-B_{S N}\right|$ to the SIT is minimized due to $\left.\Delta B\right|_{B_{S N}} \rightarrow 0$, i.e., $t$ fully scales the transition. Taking the inverse slope of $t$ plotted versus $T^{-1}$, the scaling exponent product $\nu z$ of the ENI can be determined. As shown in Figs. 3(b) and 3(c), application of the finite-size scaling theory to the ENIs may yield temperature/magnetic field-dependent $\nu z$ values. Referring to the GSPB (dotted-dashed line) in Fig. 2(b), it is found that the lower the temperature (the higher the magnetic field), the steeper the GSPB and the higher the $\nu z$ value. Ascribed to the existence of numerous ENIs, by applying the finite-size scaling analysis to any point on the GSPB, it is possible to numerically characterize the SIT at that temperature/ magnetic field, although the physical meaning of the $\nu z$ value remains an open issue in this case.

\section{Unbinding of the precursor Cooper pairs}

As shown in Fig. 1(a), the normal state thermoresistivity of the $\left.\rho_{x x}(T)\right|_{B=0 \mathrm{~T}}$ curve deviates from the $\left.\rho_{x x}(T)\right|_{B=5 \mathrm{~T}}$ curve

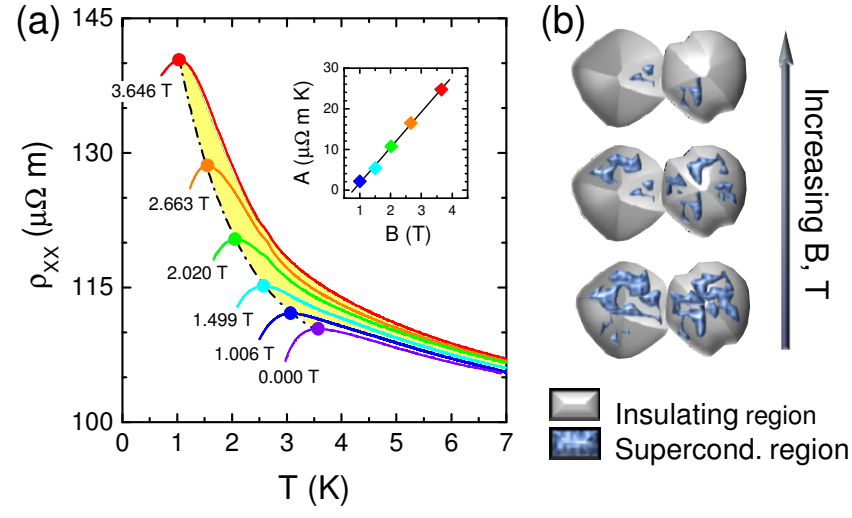

FIG. 4. (Color online) (a) Banana-shaped phase fluctuation zone highlighted in yellow. The states of $\mathrm{S} 5 \sim \mathrm{S} 0$ are indicated by filled circles from red to violet. Area in between the curve of $\left.\rho_{x x}(T)\right|_{B=0 \mathrm{~T}}$ and the curve of $\left.\rho_{x x}(T)\right|_{B \neq 0 \text { T }}$ is taken as a manifestation of the number of the unbound precursor Cooper pairs in the phase fluctuation zone. Inset: magnetic field dependence of the unbinding of the precursor Cooper pairs. (b) Precursor scenario in the phase fluctuation zone: Cooper pair-filled grains embedded in a matrix of normal state

at $T_{\text {onset }} \sim 10.16 \mathrm{~K}$ which is far above $T_{C}^{\text {mid }}=2.20 \mathrm{~K}$. This feature can be attributed to precursor pairing. ${ }^{17-20}$ Precursor Cooper pairing may be established in some intrinsic and/or extrinsic grains well above $T_{C}^{\text {mid }}$ without requiring long range phase coherence. Intergrain phase fluctuations delay the global condensation of the Cooper pairs and consequently, reduce the value of $T_{C}^{\text {mid }}$. The distinct difference between $T_{\text {onset }}$ and $T_{C}^{\text {mid }}$ implies a dispersive size distribution of the BDD grains and/or a doping inhomogeneity. Both options are very likely to occur due to the specific growth mode of CVD diamond films, with many small grains at the substrate-film interface, the number of which gradually decreases as the film becomes thicker. ${ }^{46}$

Figure 4 represents the destruction of the precursor paring by magnetic fields. As shown by the magnification in Fig. 4(a), the phase fluctuation zone, full of localized precursor Cooper pairs and hopping single quasiparticles, is enclosed by the curves of $\left.\rho_{x x}(T)\right|_{B=3.646 \mathrm{~T}},\left.\rho_{x x}(T)\right|_{B=0 \mathrm{~T}}$ and the exponentially chained $S N(N=0,1,2, \ldots, 5)$ states. Based on the finding of the intrinsic granularity in the BDD thin films studied by STM/STS measurements, ${ }^{7,10,13}$ the physical scenario of the "banana-shaped" phase fluctuation zone can be defined as shown in Fig. 4(b). Precursor superconducting grains are embedded in a normal state matrix. When a magnetic field of $B_{S N}$ is applied, the intrinsic superconducting grains may shrink and/or split. As $B_{S N}$ increases, more and more precursor Cooper pairs may become unbound. The area $A$ in between a curve of $\left.\rho_{x x}(T)\right|_{B \neq 0} \mathrm{~T}$ and the curve of $\left.\rho_{x x}(T)\right|_{B=0 \text { T }}$ can be taken as a manifestation of the number of the unbound Cooper pairs. By plotting $A$ versus $B$ as shown in the inset of Fig. 4(a), unbinding of the precursor Cooper pairs by magnetic fields is found to be characterized by a linear function.

\section{CONCLUSION}

By studying transport properties of boron-doped nanocrystalline CVD diamond, we have observed a generic phase boundary which can be attributed to the SIT in granular sys- 
tems. In the $\rho$-B plane, this phase boundary can be mapped by the intersections of certain $\rho(B)$ curves taken within well defined temperature windows. We emphasize the conceptual difference between these intersections and the TUC [quantum phase transition at $0 \mathrm{~K}$ (Ref. 41)]. It is clarified that a scaling analysis on a crossoverlike intersection may give magnetic field/temperature-dependent $\nu z$ values different from the theoretical prediction. In the $\rho$-T plane, the phase boundary separates the phase fluctuation zone from the superconducting region. Unbinding of the precursor Cooper pairs within the phase fluctuation zone is characterized by a linear function of the magnetic field.

\section{ACKNOWLEDGMENTS}

The authors are grateful for the support of the Belgian IAP, the FWO under Grant No. G.0430.07N, the INPAC Project under Project Nos. EF/05/005 and GOA/09/005, and the Methusalem Funding by the Flemish Government.

${ }^{1}$ M. Stoneham, Nature Mater. 3, 3 (2004).

${ }^{2}$ H. Ye, N. Tumilty, M. Bevilacqua, S. Curat, M. Nesladek, B. Bazin, P. Bergonzo, and R. Jackman, J. Appl. Phys. 103, 054503 (2008).

${ }^{3}$ E. A. Ekimov, V. A. Sidorov, E. D. Bauer, N. N. Mel'nik, N. J. Curro, J. D. Thompson, and S. M. Stishov, Nature (London) 428, 542 (2004).

${ }^{4}$ Y. Takano, M. Nagao, I. Sakaguchi, M. Tachiki, T. Hatano, K. Kobayashi, H. Umezawa, and H. Kawarada, Appl. Phys. Lett. 85, 2851 (2004).

${ }^{5} \mathrm{P}$. N. Volpe, J. Pernot, P. Muret, and F. Omnès, Appl. Phys. Lett. 94, 092102 (2009).

${ }^{6}$ Z. L. Wang, Q. Luo, L. W. Liu, C. Y. Li, H. X. Yang, H. F. Yang, J. J. Li, X. Y. Lu, Z. S. Jin, L. Lu, and C. Z. Gu, Diamond Relat. Mater. 15, 659 (2006).

${ }^{7}$ B. Sacépé, C. Chapelier, C. Marcenat, J. Kačmarčik, T. Klein, M. Bernard, and E. Bustarret, Phys. Rev. Lett. 96, 097006 (2006).

${ }^{8}$ T. Klein, P. Achatz, J. Kacmarcik, C. Marcenat, F. Gustafsson, J. Marcus, E. Bustarret, J. Pernot, F. Omnes, B. E. Sernelius, C. Persson, A. F. da Silva, and C. Cytermann, Phys. Rev. B 75, 165313 (2007).

${ }^{9}$ B. L. Willems, G. Zhang, J. Vanacken, V. V. Moshchalkov, S. D. Janssens, O. A. Williams, K. Haenen, and P. Wagner, J. Appl. Phys. 106, 033711 (2009).

${ }^{10}$ B. L. Willems V. H. Dao-, J. Vanacken, L. F. Chibotaru, V. V. Moshchalkov, I. Guillamón, H. Suderow, S. Vieira, S. D. Janssens, O. A. Williams, K. Haenen, and P. Wagner, Phys. Rev. B 80, 224518 (2009).

${ }^{11}$ P. Achatz, W. Gajewski, E. Bustarret, C. Marcenat, R. Piquerel, C. Chapelier, T. Dubouchet, O. A. Williams, K. Haenen, J. A. Garrido, and M. Stutzmann, Phys. Rev. B 79, 201203(R) (2009).

${ }^{12}$ W. Gajewski, P. Achatz, O. A. Williams, K. Haenen, E. Bustarret, M. Stutzmann, and J. A. Garrido, Phys. Rev. B 79, 045206 (2009).

${ }^{13}$ B. Willems, G. Zhang, J. Vanacken, V. V. Moshchalkov, H. Suderow, S. D.
Janssens, K. Haenen, and P. Wagner, "In/extrinsic granularity in superconducting boron-doped diamond," Physica C (in press).

${ }^{14}$ J. J. Mareš, P. Hubík, M. Nesládek, and J. Krištofik, Diamond Relat. Mater. 16, 921 (2007).

${ }^{15}$ O. A. Williams, O. Douhéret, M. Daenen, K. Haenen, E. Ōsawa, and M. Takahashi, Chem. Phys. Lett. 445, 255 (2007).

${ }^{16}$ B. Willems, Ph.D. thesis, Katholieke Universiteit Leuven, 2009.

${ }^{17}$ K. Kagawa, K. Inagaki, and S. Tanda, Phys. Rev. B 53, R2979 (1996).

${ }^{18}$ W. Escoffier, C. Chapelier, N. Hadacek, and J.-C. Villégier, Phys. Rev. Lett. 93, 217005 (2004).

${ }^{19}$ B. Leridon, J. Vanacken, T. Wambecq, and V. V. Moshchalkov, Phys. Rev. B 76, 012503 (2007).

${ }^{20}$ K. H. S. B. Tan, K. A. Parendo, and A. M. Goldman, Phys. Rev. B 78, 014506 (2008).

${ }^{21}$ R. W. Simon, B. J. Dalrymple, D. Van Vechten, W. W. Fuller, and S. A. Wolf, Phys. Rev. B 36, 1962 (1987).

${ }^{22}$ B. Abeles, P. Sheng, M. D. Coutts, and Y. Arie, Adv. Phys. 24, 407 (1975).

${ }^{23}$ B. Abeles, Phys. Rev. B 15, 2828 (1977).

${ }^{24}$ Y. Shapira and G. Deutscher, Phys. Rev. B 27, 4463 (1983).

${ }^{25}$ H. M. Jaeger, D. B. Haviland, B. G. Orr, and A. M. Goldman, Phys. Rev. B 40, 182 (1989).

${ }^{26}$ A. L. Efros and B. I. Shklovskii, J. Phys. C 8, L49 (1975); 9, 2021 (1976).

${ }^{27}$ N. F. Mott, J. Non-Cryst. Solids 1, 1 (1968).

${ }^{28}$ C. Quitmann, D. Andrich, C. Jarchow, M. Fleuster, B. Beschoten, G. Güntherodt, V. V. Moshchalkov, G. Mante, and R. Manzke, Phys. Rev. B 46, 11813 (1992).

${ }^{29}$ T. Sato, K. Ohashi, H. Sugai, T. Sumi, K. Haruna, H. Maeta, N. Matsumoto, and H. Otsuka, Phys. Rev. B 61, 12970 (2000).

${ }^{30}$ I. S. Beloborodov and K. B. Efetov, Phys. Rev. Lett. 82, 3332 (1999).

${ }^{31}$ I. S. Beloborodov, A. V. Lopatin, V. M. Vinokur, and K. B. Efetov, Rev. Mod. Phys. 79, 469 (2007).

${ }^{32}$ A. F. Hebard and M. A. Paalanen, Phys. Rev. Lett. 65, 927 (1990).

${ }^{33}$ K. Kim and H. J. Lee, Phys. Rev. B 54, 13152 (1996).

${ }^{34}$ S. Okuma and N. Kokubo, Phys. Rev. B 56, 410 (1997).

${ }^{35}$ N. Marković, C. Christiansen, A. M. Mack, W. H. Huber, and A. M. Goldman, Phys. Rev. B 60, 4320 (1999).

${ }^{36}$ K. A. Parendo, K. H. S. B. Tan, and A. M. Goldman, Phys. Rev. B 73, 174527 (2006)

${ }^{37}$ M. P. A. Fisher, Phys. Rev. Lett. 65, 923 (1990).

${ }^{38}$ D. A. Huse, M. P. A. Fisher, and D. S. Fisher, Nature (London) 358, 553 (1992).

${ }^{39}$ S. Okuma and N. Kokubo, Phys. Rev. B 51, 15415 (1995).

${ }^{40}$ A. Yazdani and A. Kapitulnik, Phys. Rev. Lett. 74, 3037 (1995).

${ }^{41}$ S. L. Sondhi, S. M. Girvin, J. P. Carini, and D. Shahar, Rev. Mod. Phys. 69, 315 (1997).

${ }^{42}$ N. Marković, C. Christiansen, and A. M. Goldman, Phys. Rev. Lett. 81, 5217 (1998).

${ }^{43}$ V. F. Gantmakher, M. V. Golubkov, V. T. Dolgopolov, A. A. Shashkin, and G. E. Tsydynzhapov, JETP Lett. 71, 473 (2000).

${ }^{44}$ E. Bielejec and W. Wu, Phys. Rev. Lett. 88, 206802 (2002).

${ }^{45}$ M. P. A. Fisher and G. Grinstein, Phys. Rev. Lett. 64, 587 (1990).

${ }^{46}$ C. Wild, P. Koidl, W. Müller-Sebert, H. Walcher, R. Kohl, N. Herres, R. Locher, R. Samlenski, and R. Brenn, Diamond Relat. Mater. 2, 158 (1993). 